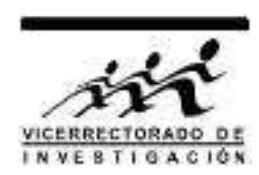

\title{
Estrategia para el estimado de los coeficientes de absorción y esparcimiento en medios participantes unidimensionales
}

\author{
M. Berrocal Tito*1, R. F. Carita Montero ${ }^{1}$, J. A. Bravo ${ }^{1}$ y A. J. da Silva Neto ${ }^{2}$ \\ ${ }^{1}$ Facultad de Ciencias Físicas, Universidad Nacional Mayor de San Marcos, Lima, Perú \\ ${ }^{2}$ Departamento de Ing. Mecánica y Energía, Universidade do Estado do Rio de Janeiro, Rio de Janeiro, Brasil
}

Recibido 30 marzo 2014 - Aceptado 15 junio 2014

\begin{abstract}
En este trabajo se presenta una estrategia para la estimación de los coeficientes de absorción y de dispersión en medios participantes de una dimensión. Se considera medios con el coeficiente de absorción en el intervalo [0.1-1.0] y el coeficiente de esparcimiento entre [0.1-1.0]. El problema directo fue resuelto con los métodos de ordenadas discretas y diferencias finitas. Para resolver el problema inverso se presenta la siguiente estrategia que consiste en (a) encontrar la estimativa del coeficiente de absorción considerando el coeficiente de esparcimiento con un valor aproximado a 0.01 , (b) se estima el coeficiente de esparcimiento utilizando el valor de coeficiente de absorción ya calculado. La función error es definida como la diferencia entre el valor medido por el detector y el calculado por el problema directo. El algoritmo usado para la solución es minimizar la distancia de Bregman sujeto a la función error. La distancia de Bregman fue construida con la función relacionada a la entropía de Havdra-Charvát.

Casos de prueba con ruido aleatorio hasta $2 \%$ en los datos medidos son presentados. Con la finalidad de encontrar la mejor estimativa adoptamos como criterio de comparación de los resultados el error porcentual medio cuadrático.

Palabras claves: problema inverso, distancia de Bregman, transferencia de calor, entropía de HavdraCharvát.
\end{abstract}

\section{Strategy for the estimation of the scattering and absorption coefficients in one-dimensional participating media}

In this work a strategy for the estimation of absorption and scattering coefficients in one-dimensional participating media is presented. Media are considered with the absorption coefficient in the range $[0.1$ to 1.0] and the scattering coefficient between [0.1-1.0]. The direct problem was solved with the discrete ordinates and finite difference methods. In order to solve the inverse problem the following strategy consists of (a) find the absorption coefficient considering the scattering coefficient with an approximate value. 0.01, (b) find the scattering coefficient value using the absorption coefficient estimated in (a). The error function is defined as the difference between the measured value by the detector and the calculated by the direct problem. The algorithm used for the solution is to minimize the Bregman distance subject to the error function. Bregman distance was constructed with a related function to the entropy of Havdra-Charvát. Cases random noise tests to $2 \%$ in the measured data are presented. In order to find the best estimate we adopt as a criterion for comparison of the relative standard quadratic error.

Keywords: inverse problem, Bregman distance, heat transfer, entropy Havdra - Charvát.

Diferentes tipos de radiación, tales como partículas neutras, cargadas y fotones han sido usados para a identificación de objetos en la industria, en ensayos no destructivos, así como en la medicina, para diagnóstico y terapia. En muchas de las técnicas usadas para la reconstrucción de imágenes, el esparcimiento en el medio no es considera- do, llevando a problemas lineales de solución numérica más simple, pero que poseen aplicaciones tecnológicas relevantes. Dos ejemplos de esta simplificación son la tomografía computadorizada (Computerized Tomography, CT) [1], y la tomografía computadorizada por emisión de fotón único (Single Photon Emission Computerized Tomography,

${ }^{*}$ mabet99@yahoo.com 
SPECT), [2].

Para los casos en que el esparcimiento no puede ser despreciado, tal como en la tomografía óptica infrarroja (Near Infrared Optical Tomography, NIROT), el modelo de reconstrucción de la imagen es más complejo y es no lineal. El análisis de la tomografía se encuentra en el mismo contexto del transporte de partículas neutras en los reactores nucleares y en la transferencia de calor por radiación térmica en los medios participantes, donde los fenómenos físicos relevantes como la absorción, emisión y esparcimiento son modelados por la ecuación de transferencia radiativa [3]. Este trabajo es parte de un proyecto para estimar los coeficientes de absorción y esparcimiento en medios de 2 dimensiones (2D) a partir de la solución unidimensional (1D). El método de ordenadas discretas tiene como desventaja la presencia del efecto rayo, cuando es empleado en un medio $2 D[4,5]$. El efecto rayo no se presenta en una dimensión. Siendo este el motivo que se propone la aproximación 1D al 2 D [6].

\section{El Problema Directo}

La ecuación de transporte de radiación en coordenadas cartesianas para un medio unidimensional en régimen permanente, considerando simetría azimutal, sin dependencia espectral, en un medio isotrópico y sin fuentes está dado por

$$
\mu_{l} \frac{d I_{l}(x)}{d x}+\sigma_{t}(x) T_{l}(x)=Q_{l}(x),
$$

donde

$$
Q_{l}=\sum_{m=1}^{L_{o}} \frac{\sigma^{s}(x)}{4 \pi} w_{m} I_{m}(x),
$$

con $l=1,2, \ldots, L_{0} ; 0 \leq x \leq L \mathrm{y}-1 \leq \mu_{1} \leq 1$, además $I_{l}(x)$ es la intensidad de la radiación, $x$ es la variable espacial, $\mu_{l}$ es el coseno de la dirección de propagación con el eje $x$ (coseno del ángulo polar), $\sigma^{s}(x)$ es el coeficiente de esparcimiento, $\sigma^{a}() x$ es el coeficiente de absorción, $\sigma_{t}(x)=\sigma^{a}(a)+\sigma^{s}(x)$ es el coeficiente de extinción total.

La discretización del dominio angular se realiza en lo ángulos sólidos $\Omega_{l}$, donde $l=1,2, \ldots, L_{0}$, y $w_{l}$ es el peso de la cuadratura asociada a la dirección $l$. El ángulo sólido $\Omega_{l}$ alrededor del eje $x$ va ser representada por los cosenos directores $\mu_{l}$ [3].

La Figura 1, presenta el problema físico a resolver en una placa larga de espesor $L_{x}$. El dominio espacial es discretizado en $E$ intervalos regulares $\Delta x$, como se aprecia en la Figura 1, la longitud del medio $L_{x}$ es igual a $L_{x}=E \Delta x$. Producto de la discretización cada segmento espacial va ser identificado por un subíndice $e$. El centroide del segmento $e$ (ver Figura 1) es determinado por

$$
x_{e}=(2 e-1) \frac{\Delta x}{2} .
$$

Considerando $I w_{e}$ e $I e_{e}$ los flujos que ingresan por los puntos de frontera del segmento $x_{e}$, Figura 1, calculamos la intensidad $I_{e}$ en el centro de cada segmento $x_{e}$, mediante

$$
\Delta x=x_{e+1}-x_{e} \quad \text { y } \quad \Delta I_{l, e}=I e_{l, e}-I w_{l, e} .
$$

$\mu_{l}$ está dentro de los intervalos $-1 \leq \mu_{l} \leq 1$. Entonces el valor positivo o negativo de $\mu_{l}$ describe 2 cuadrantes, Figura 2, en los cuales la radiación puede ser propagada o sufrir esparcimiento.
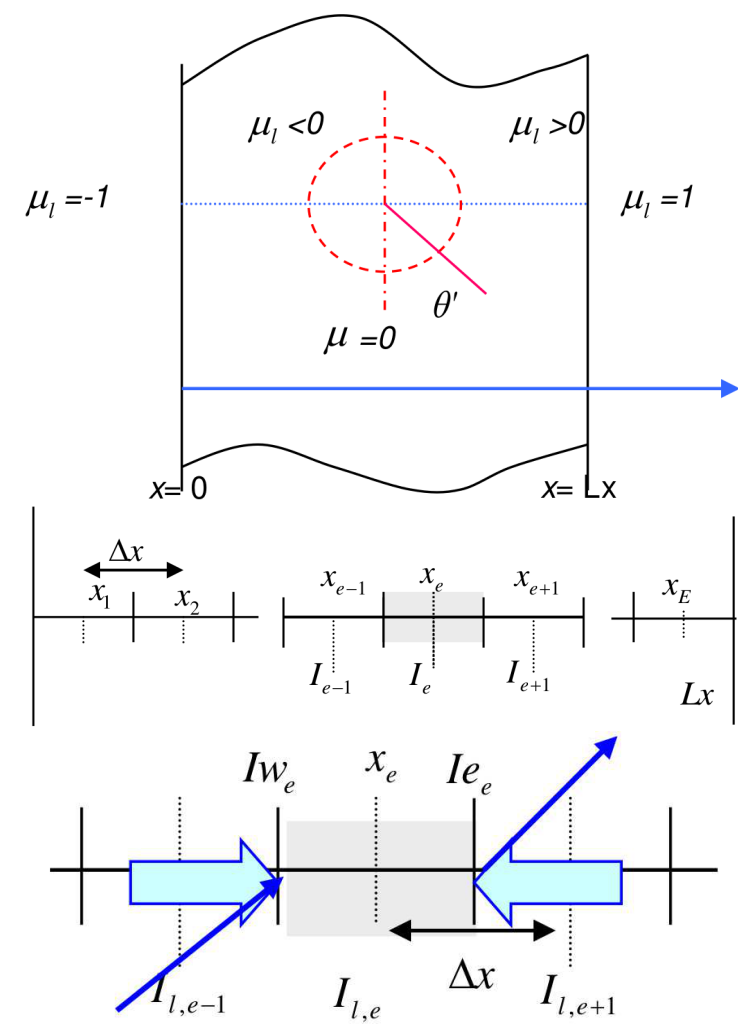

Figura 1: Medio en una dimensión (arriba). Discretización del dominio espacial en diferencias finitas (medio). Aproximación por diferencias (abajo).

Aproximando las derivadas por diferencias finitas en la ecuación (1) obtenemos

$$
\mu_{1} \frac{\Delta I_{l, e}}{\Delta x}+\sigma_{t, e} I_{l, e}=Q_{l, e} ; l=1,2, \ldots, L_{0},
$$

y reemplazando la ecuación (3) en la ecuación (4), obtenemos

$$
\mu_{1} \frac{I e_{l, e}-I w_{l, e}}{\Delta x}+\sigma_{t, e} I_{l, e}=Q_{l, e} ; l=1,2, \ldots, L_{0},
$$

el valor de la intensidad $I_{l, e}$ puede ser aproximado a

$$
I_{l, e}=\gamma I e_{l}+(1-\gamma) I w_{l, e} \text { con } \gamma=0.5
$$

multiplicando la ecuación (5) por $\gamma \Delta x$, obtenemos

$$
\mu_{l}\left(\gamma I e_{l, e}-\gamma I w_{l, e}\right)+\gamma \Delta x \sigma_{t, e} I_{l, e}=\gamma \Delta x Q_{l, e}
$$


donde $l=1,2, \ldots, L_{0}$.

La intensidad $I_{l, e}$ puede viajar por dos cuadrantes, ver Figura 2. El cuadrante $I$ indica una marcha para el este, con los cosenos directores $\mu_{l}>0$. El cuadrante $I I$ indica una marcha para el oeste con $\mu_{l}<0$. La intensidad $I_{l, e}$ para cada marcha es descrita a continuación.

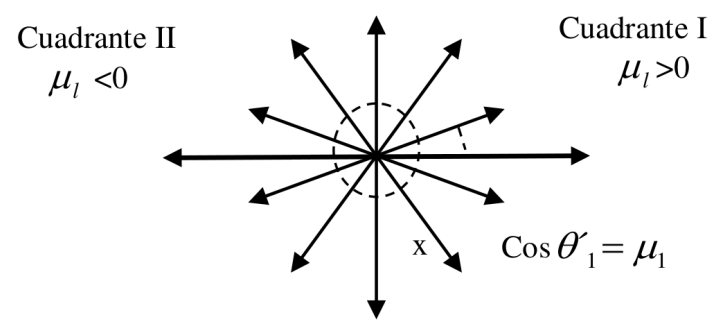

Figura 2: Cuadrantes descritos por los cosenos directores $\mu_{l}$.

El cuadrante I, ocurre para $\mu_{l}>0$, la dirección de $I_{l, e}$ está en la dirección de $I w$, luego

$$
I e_{l, e}=2 I_{l, e}-I w_{l, e},
$$

reemplazando la ecuación 8 en la ecuación 7 , se tiene

$$
I_{l, e}=\frac{2 \mu_{1} I w_{l, e}+\Delta x Q_{l, e}}{2 \mu_{l}+\sigma_{t, e} \Delta x},
$$

donde $l=1,2, \ldots, L_{0}$ y $e=1,2, \ldots, E$.

Considerando las condiciones de frontera entre los elementos de área adyacentes. Si $e<E$ tenemos que $I w_{e+1}=I e_{e}$.

En el cuadrante II, sucede para $\mu_{l}<0$, la dirección de $I_{l, e}$ está en la dirección de $I e$, luego

$$
I w_{l, e}=2 I_{l, e}-I e_{l, e},
$$

reemplazando la ecuación 10 en la ecuación 7 , se tiene

$$
I_{l, e}=\frac{-2 \mu_{l} \cdot I e_{l, e}+\Delta x Q_{l, e}}{-2 \mu_{l}+\Delta x \sigma_{t, e}}
$$

donde $l=1,2, \ldots, L_{0}$ y $e=E, E-1, \ldots, 1$; considerando las condiciones de frontera entre los elementos de área adyacentes.

Si $e>1$, tenemos

$$
I e_{e-1}=I w_{e} .
$$

Para determinar $I_{e}$ necesitamos de $Q_{l, e}$, observamos que $Q_{l, e}$ depende de $I_{l, e}$. Por lo que es necesario utilizar un procedimiento iterativo para la obtención de una solución aproximada para $I_{l, e}$. Sea $h$ un contador de iteraciones. La ecuación 9 puede ser escrita como

$$
I_{l, e}^{h}=\frac{2 \mu_{l} I w_{l, e}^{h}+\Delta x Q_{l, e}^{h-1}}{2 \mu_{l}+\sigma_{t, e} \Delta x},
$$

donde

$$
Q_{l, e}^{h-1}=\sum_{m=1}^{L-0} \frac{\sigma_{S, e}}{4 \pi} w_{m} \cdot I_{m, e}^{h-1} \text { y } l=1,2, \ldots L_{0},
$$

cualquier intensidad medida de un detector $q_{e}$ posicionado en un elemento $e$ puede ser escrita como una función de la forma

$$
q_{e, \text { medido }}=F_{k}\left(\sigma^{a}, \sigma^{s}\right) .
$$

\section{El problema inverso}

Sean los vectores $\sigma^{a}$ y $\sigma^{s}$ formado por coeficiente de absorción y del coeficiente de esparcimiento en cada elemento de área, que son las incógnitas del sistema,

$$
\sigma^{a}=\left(\sigma_{1}^{a}, \sigma_{2}^{a}, \ldots, \sigma_{E}^{a}\right) \quad \sigma^{s}=\left(\sigma_{1}^{s}, \sigma_{2}^{s}, \ldots, \sigma_{E}^{s}\right) .
$$

Siendo $E$ el número de elementos o segmentos en que esta particionado el medio. Las intensidades medidas experimentalmente se denotan por $q_{\text {med }}^{k}$ con $k=1, \ldots, K$, donde $K$ es el número total de datos experimentales.

La función error $G_{k}\left(\sigma^{a}, \sigma^{s}\right)$, es definida como la diferencia entre $q_{\text {med }}^{k}$ y la intensidad calculada por el problema directo, $F_{k}\left(\sigma^{a}, \sigma^{s}\right)$,

$$
G_{k}\left(\sigma^{a}, \sigma^{s}\right)=q_{\text {med }}^{k}-F_{k}\left(\sigma^{a}, \sigma^{s}\right) .
$$

El método de máxima entropía está basado en la minimización de la distancia de Bregman $D$, restringida a la función error,

$$
\operatorname{mín} D\left(\sigma, \sigma_{0}\right)=G(\sigma)
$$

siendo la distancia de Bregman [7] construida con una función convexa $\eta$,

$$
D\left(\sigma, \sigma_{0}\right)=\eta(\sigma)-\eta\left(\sigma_{0}\right)-\left\langle\nabla \eta\left(\sigma_{0}\right), \sigma-\sigma_{0}\right\rangle,
$$

con

$$
\left\langle\nabla \eta\left(\sigma_{0}\right), \sigma-\sigma_{0}\right\rangle=\left.\sum_{e=1}^{E} \frac{\partial \eta}{\partial \sigma_{e}}\right|_{\sigma=\sigma_{0}}\left(\sigma_{e}-\sigma_{0 e}\right),
$$

siendo $\sigma_{0}$ una información a priori de valor $\sigma$.

En este trabajo para la construcción de la distancia de Bregman se va utilizar la función convexa relacionadas a las entropías de Havdra-Charvát [8], definida por

$$
\eta_{r}(\sigma)=\sum_{e=1}^{E} \frac{\sigma_{e}-\sigma_{e}^{e}}{1-r}, \text { con } r>0 .
$$

Para cada valor de $r$ se tiene un $\eta$ diferente, por tanto una distancia de Bregman diferente que puede ser usada en la construcción del lagrangiano

$$
L_{r}\left(\sigma, \sigma_{o}, \lambda\right)=D\left(\sigma, \sigma_{0}\right)+\sum_{k=1}^{K}\left[\lambda_{k} G_{k}(\sigma)\right]
$$

donde $\lambda_{k}$ son los multiplicadores de Lagrange, donde $k=1, \ldots, K$; observe que la Ec. 20 presenta como incógnita el vector $\sigma$ formado por los $\sigma_{e}$ donde $e=1,2, \ldots, E$. Lo que da un total de $(E+K)$ incógnitas.

De las Ecs. 18, 19 y 20 obtenemos el lagrangiano 


$$
L_{r}\left(\sigma, \sigma_{0}, \lambda\right)=\frac{1}{1-r}\left[\sum_{e=1}^{E}\left(\sigma_{e}-\sigma_{e}^{r}\right)-\sum_{e=1}^{E}\left(\sigma_{0 e}-\sigma_{0 e}^{r}\right)-\sum_{e=1}^{E}\left(1-r \sigma_{0 e}^{r-1}\right)\left(\sigma_{e}-\sigma_{0 e}\right)\right]+\sum_{k=1}^{K}\left[\lambda_{k} G_{k}(\sigma)\right] .
$$

Si $r \rightarrow 1$,

$$
L_{1}\left(\sigma, \sigma_{0}, \lambda\right)=-\left[\sum_{e=1}^{2 E}\left(\sigma_{e}-\sigma_{0 e}-\sigma_{e} \ln \frac{\sigma_{e}}{\sigma_{0 e}}\right)+\sum_{k=1}^{K}\left[\lambda_{k} G_{k}(\sigma)\right]\right]
$$

cuando $r=1$, la función $\eta$ coincide con la entropía de Shannon [11].

Para el estimado de las incógnitas $\sigma^{a}$ y $\sigma^{s}$ debemos resolver las Ecs. (21) o (22) según sea el caso, buscamos el punto crítico del lagrangiano, es decir, buscamos el $\sigma$, que haga tender a cero el lagrangiano. Para eso, igualamos a cero as derivadas del lagrangiano con respecto a todas sus incógnitas,

$$
\begin{aligned}
& \frac{\partial}{\partial \sigma_{e}} L\left(\sigma, \sigma_{0}, \lambda\right)=0, \quad e=1,2, \ldots, E, \\
& \frac{\partial}{\partial \lambda_{k}} L\left(\sigma, \sigma_{0}, \lambda\right)=0, \quad k=1,2, \ldots, K .
\end{aligned}
$$

Las ecuaciones 23 y 24 ofrecen $(E+K)$ ecuaciones, por lo que ahora tenemos un sistema formado de $(E+K)$ incógnitas y $(E+K)$ ecuaciones; para resolver este sistema de ecuaciones, fue escrito un código en MATLAB. Este $m$-file hace uso de las herramientas encontradas en el Toolbox de optimización del MATLAB. La minimización de este sistema fue obtenida con los algoritmos Quasi-Newton y busca lineal.

\section{Resultados}

Nuestro objetivo es encontrar las mejores condiciones como son el mejor $r$, los mejores valores iniciales y el mínimo número de detectores a ser empleado, para estimar el coeficiente de esparcimiento considerando datos con ruido aleatorio. En todos los casos se considera que la intensidad de la fuente es igual a 1.

En la Fig. 3, los detectores $q_{E}^{t}$ y $q_{1}^{t}$, miden los flujos transmitidos y los detectores $q_{1}^{r}$ y $q_{E}^{r}$ los flujos reflejados. Las intensidades de los detectores son calculadas por las ecuaciones

$$
\begin{aligned}
q_{E}^{t} & =\sum_{l=1, \mu_{l}>0}^{L_{0}} w_{l} I e_{l, E} \mu_{l}, \\
q_{1}^{r} & =\sum_{l=1, \mu_{l}<0}^{L_{0}}-w_{l} I w_{l, 1} \mu_{l}
\end{aligned}
$$

$$
\begin{gathered}
q_{1}^{t}=\sum_{l=1, \mu_{l}>0}^{L_{o}} w_{l} I w_{l, 1} \mu_{l}, \\
q_{E}^{r}=\sum_{l=1, \mu_{l}<0}^{L_{o}}-w_{l} I e_{l, E} \mu_{l} .
\end{gathered}
$$
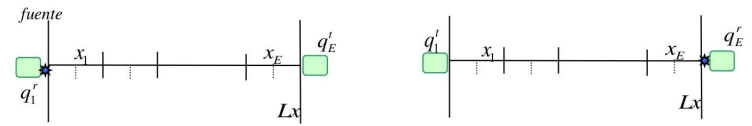

Figura 3: Geometría usada para la posición de los detectores

Es muy importante considerar la forma de cómo los datos, medidos en los detectores, van a ser empleados, pues ellos también influyen en el error para la estimación de $\sigma^{a}$ y $\sigma^{s}$.

Existen dos modos para ello, el primero, para la estimación del coeficiente de absorción se consideran las medidas de 4 detectores, los transmitidos $q_{1}^{t}$ y $q_{E}^{t}$ y los reflejados $q_{1}^{r}$ y $q_{E}^{r}$. Mientras que en el segundo modo, para la estimación del coeficiente de esparcimiento se consideran solo las medidas de los detectores reflejados $q_{1}^{r}$ y $q_{E}^{r}$.

Buscando un número que nos indique la calidad de las estimaciones de las incógnitas, definimos el error porcentual medio

$$
\mathrm{EP}=100 \% \sqrt{\sum_{e=1}^{E}\left(\frac{\sigma_{e, \text { exacto }}-\sigma_{e, \text { calculado }}}{\sigma_{e, \text { exacto }}}\right)^{2}}
$$

donde el subíndice $e$ indica un elemento discretizado del dominio y $E$ el número total de segmentos.

La validación de nuestro programa fue realizada comparando nuestros resultados con los resultados publicados $[9,10]$. La Tabla 1, muestra el proceso de comparación.

La tabla 2 presenta el conjunto de ángulos y pesos, LSH $S_{10}$, que discretiza el espacio angular, empleado en este trabajo. Se observa que el método empleado en este trabajo y el método de elementos finitos combinado con el LSH $S_{10}$ son los valores más próximos a los valores exactos. 


\begin{tabular}{cccccc}
\hline$\sigma_{t}$ & $\begin{array}{c}\text { Valores } \\
\text { exactos }\end{array}$ & $\begin{array}{c}\text { Armónicos } \\
\text { esféricos } P_{l}\end{array}$ & $\begin{array}{c}\text { Elementos Finitos } \\
\text { LSH } S_{10}\end{array}$ & $\begin{array}{c}\text { Diferencias Finitas } \\
\text { LSH } S_{10}\end{array}$ & $\begin{array}{c}\text { Diferencias Finitas } \\
\text { Gauss-Legendre } S_{20}\end{array}$ \\
\hline 0.0 & 1.0000 & 1.0000 & 1.0000 & 1.0000 & 1.0000 \\
0.1 & 0.9157 & 0.9302 & 0.9138 & 0.9138 & 0.9145 \\
0.5 & 0.7040 & 0.7273 & 0.7027 & 0.7026 & 0.7026 \\
1.0 & 0.5532 & 0.5714 & 0.5530 & 0.5529 & 0.5517 \\
5.0 & 0.2077 & 0.2105 & 0.2076 & 0.2078 & 0.2070 \\
\hline
\end{tabular}

Tabla 1: Valores calculados de $q_{\text {med }}$ para el problema propuesto por Fiveland [10].

\begin{tabular}{cccccc}
\hline $\begin{array}{c}\text { Punto } \\
\text { Número }\end{array}$ & $\begin{array}{c}\text { Ordenadas } \\
\mu_{l}\end{array}$ & $\begin{array}{c}\text { Pesos } \\
w_{l}\end{array}$ & $\begin{array}{c}\text { Punto } \\
\text { Número }\end{array}$ & $\begin{array}{c}\text { Ordenadas } \\
\mu_{l}\end{array}$ & $\begin{array}{c}\text { Pesos } \\
w_{l}\end{array}$ \\
\hline 1 & 0.1372719 & 2.0122 & 6 & -0.1372719 & 2.0122 \\
2 & 0.5046889 & 2.1071 & 7 & -0.5046889 & 2.1071 \\
3 & 0.7004129 & 0.5990 & 8 & -0.7004129 & 0.5990 \\
4 & 0.8523177 & 1.1872 & 9 & -0.8523177 & 1.1872 \\
5 & 0.9809754 & 0.3778 & 10 & -0.9809754 & 0.3778 \\
\hline
\end{tabular}

Tabla 2: Puntos de colocación y peso de la cuadratura $\operatorname{LSH} S_{10}, L_{0}=10$.

La estimación del coeficiente de absorción empleando la función

$$
\eta_{r}\left(\sigma_{e}\right)=\frac{\left(\sigma_{e}-\sigma_{e}^{r}\right)}{1-r}
$$

en la construcción de la distancia de Bregman se comienza encontrando el mejor valor de $r$. El mejor $r$ va a depender de la información de los valores iniciales, del ruido en las medidas de los detectores y del número de detectores a ser empleados. Después de muchas pruebas se observó que $\sigma^{a, s} \sim 10^{-6}$ proporcionan mejores resultados para minimizar el sistema de Ecs. (23) y (24), cuando no se tiene conocimiento alguno de los valores de las incógnitas.

Aquí se presentan dos casos para encontrar el mejor $r$ para estimar el $\sigma^{a}$ y $\sigma^{s}$.

En el primer caso, se obtiene el mejor $r$ para la estimación de $\sigma^{a}$ considerando un valor de $\sigma^{s}$ conocido para un medio homogéneo dado. Para el cual empleamos los cuatro detectores que se consideran en el primer modo con valor un inicial $\sigma^{a, 0}=10^{-6}$.

La Fig. 4(a) presenta los errores porcentuales en la estimación del coeficiente de absorción, AC, iguales a 0.1 , $0.2, \ldots, 1.0$, usando datos de los detectores del primer modo y sin ruido aleatorio. En la Fig. 4(b) se tiene $r$ entre 1 a 20 . Obsérvase que $r=10$ es un buen valor para ser utilizado en la construcción de la distancia de Bregman.

En la Fig. 5(a) y 5(b) se observan los resultados de los errores porcentuales en la estimación de los coeficientes de absorción empleando valores de $r \in[0.0,1.0]$ y $r \in[1.0,10.0]$ con datos a los cuales se han adicionado ruido aleatorio hasta el $2 \%$, respectivamente.

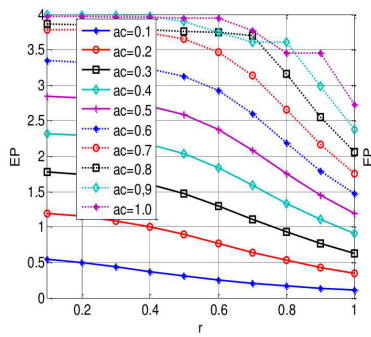

(a)

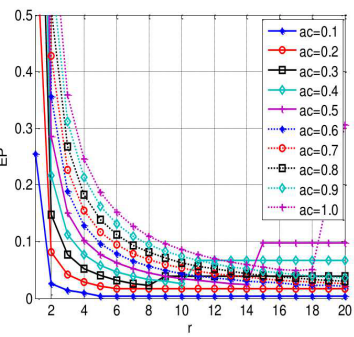

(b)
Figura 4: Error porcentual en la estimación de $\sigma^{a}$ empleando diferentes valores $r$. Datos exactos.

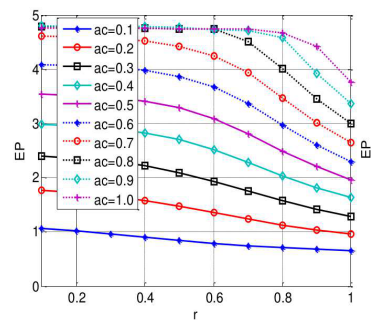

(a)

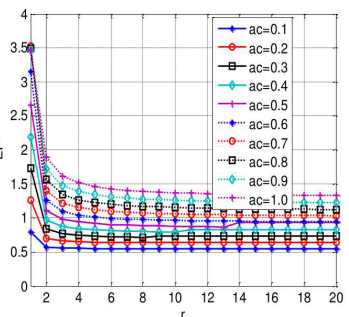

(b)
Figura 5: Error porcentual al estimar los coeficientes de absorción, $\sigma^{a}$, empleando (a) $r=[0.1-1]$, y (b) $r=[1-20]$, con $2 \%$ de error en los datos.

El segundo caso es la estimación de $\sigma^{s}$ con el $\sigma^{a}$ conocido para un medio determinado, para encontrar el mejor $r$ se considera dos posiblidades, la primera ocurre cuando $\sigma^{s}>0.1$, aquí se emplea los detectores reflejados en el segundo modo y un valor de los coeficientes de esparcimiento inicial de $\sigma^{s, 0}=10^{-6}$. La Fig.6, presenta el error 
porcentual en la estimación del coeficiente de esparcimiento (sc) en el intervalo de $[0.1-1.0]$, empleando diferentes valores de $r$ que varían entre 1 y 20. En la Fig. 6(a) se emplearon datos exactos y en la Fig. 6(b) se emplearon datos con ruido aleatorio hasta de $2 \%$. En este caso, un buen resultado se obtuvo con $r=6$, para datos exactos y con ruido aleatorio. La segunda posibilidad ocurre cuando $\sigma^{s} \leq 0.1$, en la cual se emplean solo detectores reflejados y un valor de los coeficientes de esparcimiento inicial de $\sigma^{s, o}=10^{-6}$. La Fig. 7, presenta el error porcentual al estimar el coeficiente de esparcimiento (sc) en el intervalo de $[0.01-0.1]$, empleando diferentes valores de $r$ que varían entre 1 y 20. En la Fig. 7(a) se emplearon los datos exactos y en la Fig. 7(b) se grafican los datos con ruido aleatorio de hasta $2 \%$. En este caso un buen resultado se obtuvo con $r=3$, para datos exactos y con ruido aleatorio.

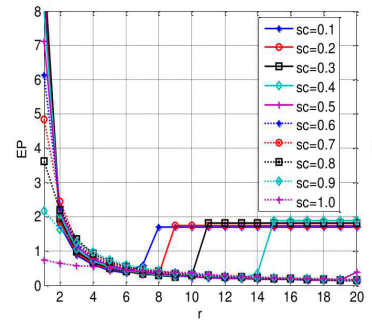

(a) Datos exactos

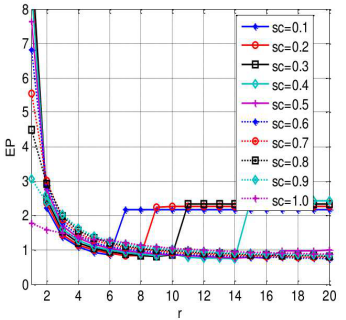

(b) ruido aleatorio $<2 \%$
Figura 6: Error porcentual en la estimación de $\sigma^{s}>0.1$, con diferentes valores de $r$.

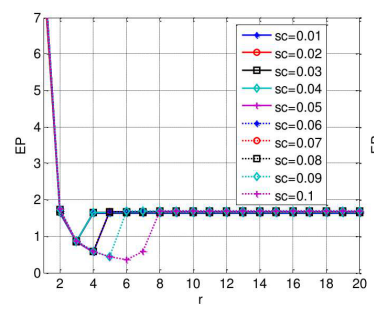

(a) datos exactos

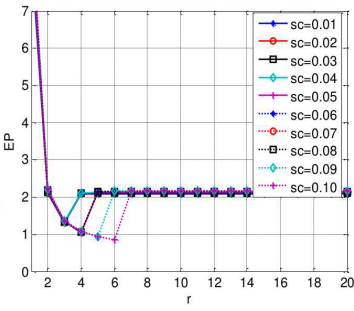

(b) ruido aleatorio $<2 \%$
Figura 7: Error porcentual en la estimación de $\sigma^{s}<0.1$, con diferentes valores de $r$.

Ahora calculamos el parámetro $r$, cuando los valores iniciales de $\sigma^{a}$ y de $\sigma^{s}$ están entre el 10 y $15 \%$. Comparando los EP de las estimaciones de $\sigma^{a}$ y $\sigma^{s}$, obtenidas variando los valores de $r$ entre 0 y 20 , encontramos que el mejor $r$ fue para $r=4$.

En todo este proceso, el objetivo es determinar un algoritmo que nos proporcione una buena estimación de los coeficientes de absorción y esparcimiento al emplear datos con ruido aleatorio que simulen las medidas reales. Es posible encontrar los valores de $r$, que ofrecen muy buenos resultados cuando usamos datos exactos, sin embargo, cuando le adicionamos ruido aleatorio o utilizamos datos reales experimentales estos algoritmos se vuelven inestables e inciertos como sucede en el caso que presentamos a continuación.

En el experimento simulamos los datos exactos de cuatro detectores y realizamos las estimaciones para $\sigma^{s}>0.1$. En la Fig. 8(a) y (b) mostramos que los errores porcentuales son muy pequeños considerando que los datos mostrados en la Fig. 8(b) son datos incluyendo un ruido aleatorio menor a $0.5 \%$. Pero estos errores porcentuales son muy superiores a los errores porcentuales obtenidos en la Fig. 7 (b) con ruido aleatorio de hasta $2 \%$.

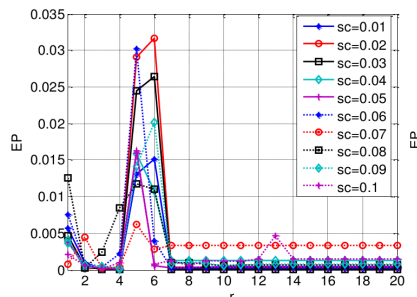

(a)

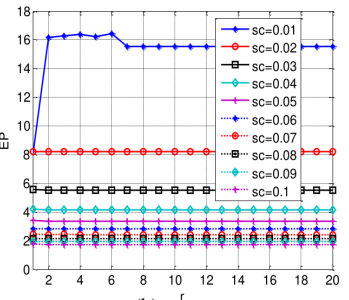

Figura 8: Error porcentual obtenido con cuatro detectores en la estimación de $\sigma^{s}<0.1$

Los errores porcentuales de los dos primeros casos para estimar $\sigma^{a}$ y $\sigma^{s}$, se disminuye si utilizamos los $\sigma^{a}$ y $\sigma^{s}$ obtenidos después de una corrida, como valores iniciales para resolver nuevamente el sistema de ecuaciones dados por la Ecs. (23) y (24), este proceso se repite de manera iterativa tal como se describe a continuación.

Para la estimación de $\sigma^{a}$ y $\sigma^{s}$, para un medio homogéneo, siendo ambos desconocidos procedemos a (1) minimizar las Ecs. (23) y (24) para la estimación de $\sigma^{a}$ empleando los datos de cuatro detectores y considerando $\sigma^{a, 0}=10^{-6}, \sigma^{s}=0.001, r=10$ y calculamos

$$
\bar{\sigma}^{a}=\sum_{e=1}^{E} \sigma_{e}^{a} / E ;
$$

(2) minimizamos las Ecs. (23) y (24) para la estimación de $\sigma^{s}$ empleando los datos de los detectores reflejados, con $\sigma^{s, 0}=10^{-6}, \bar{\sigma}^{a}$ obtenido del proceso anterior, $r=6$ y calculamos

$$
\bar{\sigma}^{s}=\sum_{e=1}^{E} \sigma_{e}^{s} / E
$$

(3) minimizamos nuevamente las Ecs. (23) y (24) para la estimación de $\sigma^{a}$ empleando los datos de los cuatro detectores, con $\sigma^{a, 0}=\bar{\sigma}^{a}, \bar{\sigma}^{s}$ obtenido del proceso anterior, $r=4$ y calculamos

$$
\bar{\sigma}^{a}=\sum_{e=1}^{E} \sigma_{e}^{a} / E ;
$$

(4) minimizamos las Ecs. (23) y (24) para la estimación de $\sigma^{s}$ empleando los datos de los detectores reflejados, con 
$\sigma^{s, 0}=\bar{\sigma}^{s}$, con $\bar{\sigma}^{a}$ obtenido del paso anterior, con $r=4$ y calculando

$$
\bar{\sigma}^{s}=\sum_{e=1}^{E} \sigma_{e}^{s} / E ;
$$

los procesos (3) y (4) se repiten iterativamente hasta obtener los errores porcentuales razonablemente bajos.

En la Fig. 9, se presenta los errores porcentuales de los procesos 1 hasta el proceso 6 , para el estimado de los coeficientes $\sigma^{s}$ y $\sigma^{s}$, cuando se añade un ruido aleatorio de hasta $2 \%$ en los datos de los detectores.
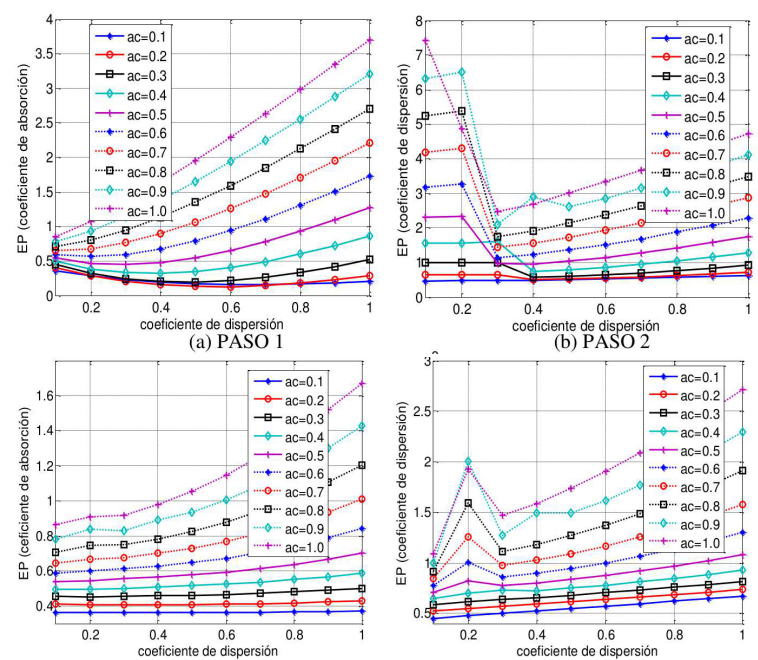

(c) $\mathrm{PASO} 3$

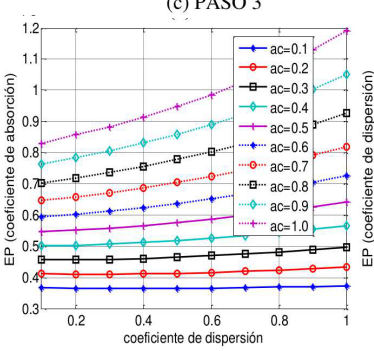

(c) PASO 5

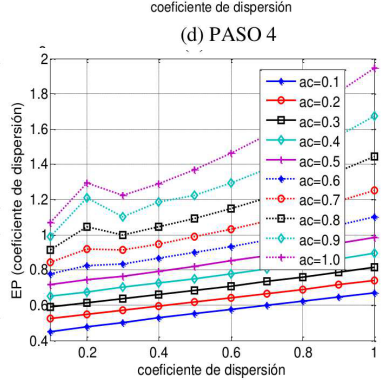

(d) PASO 6
Figura 9: Errores porcentuales obtenidos en la estimación de $\sigma^{a}$ y $\sigma^{s}$, ambos obtenidos entre $0.1-1.0$ usando datos con ruido aleatorio menores de $2 \%$.

Finalmente, procedemos a estimar los coeficientes $\sigma^{a}$ y $\sigma^{s}$ para un medio formado por dos capas. El medio está esquematizado en la Fig. 10, donde las dos capas tiene una interface común y estimamos los coeficientes de absorción $\sigma_{1}^{a}$ en el medio $1, \sigma_{2}^{a}$ en el medio 2 y el coeficiente de esparcimiento $\sigma^{s}$ para ambos medios.

En este caso el procedimiento de cálculo iterativo de la estimación de los coeficientes de absorción y esparcimiento consiste en (1) minimizar las Ecs. (23) y (24) para la estimación de $\sigma^{a}$ empleando los datos de los cuatro detectores, con $\sigma^{a, 0}=10^{-6}$, con $\sigma^{s}=0.09$, con $r=10 \mathrm{y}$ calculamos los dos coeficientes de los medios 1 y 2

$$
\begin{array}{r}
\bar{\sigma}_{1}^{a}=\sum_{e=1}^{E_{1}} \frac{\sigma_{e}^{a}}{E_{1}}, \\
\bar{\sigma}_{2}^{a}=\sum_{e=E_{1}+1}^{E_{2}} \frac{\sigma_{e}^{a}}{E_{2}-E_{1}} ;
\end{array}
$$

(2) minimizar las Ecs. (23) y (24) para la estimación de $\sigma^{s}$ empleando los datos de los detectores reflejados con $\sigma^{s, 0}=10^{-6}, \bar{\sigma}_{1}^{a}$ y $\bar{\sigma}_{2}^{a}$ obtenidos en proceso anterior, con $r=6$ y calculando

$$
\bar{\sigma}^{s}=\sum_{e=1}^{E_{2}} \sigma_{e}^{s} / E ;
$$

(3) minimizar las Ecs. (23) y (24) para la estimación de $\sigma^{a}$ empleando los datos de todos los detectores con $\sigma^{a, 0}=10^{-6}, \bar{\sigma}^{s}$ obtenido del paso anterior, $r=4$ y calculando nuevamente

$$
\begin{array}{r}
\bar{\sigma}_{1}^{a}=\sum_{e=1}^{E_{1}} \frac{\sigma_{e}^{a}}{E_{1}}, \\
\bar{\sigma}_{2}^{a}=\sum_{e=E_{1}+1}^{E_{2}} \frac{\sigma_{e}^{a}}{E_{2}-E_{1}} ;
\end{array}
$$

(4) minimizar las Ecs. (23) y (24) para la estimación de $\sigma^{s}$ empleando los datos de los detectores reflejados con $\sigma^{s, 0}=10^{-6}$, con $\bar{\sigma}_{1}^{a}$ y $\bar{\sigma}_{2}^{a}$ obtenidos en el proceso anterior, con $r=4$ y luego calculamos

$$
\bar{\sigma}^{s}=\sum_{e=1}^{E_{2}} \sigma_{e}^{s} / E
$$

a partir de aquí los procesos (3) y (4) se repiten iterativamente hasta alcanzar un mínimo en los errores porcentuales. Para todos los procesos hemos considerando un ruido aleatorio menor a $1 \%$ añadidos a las medidas de los detectores.

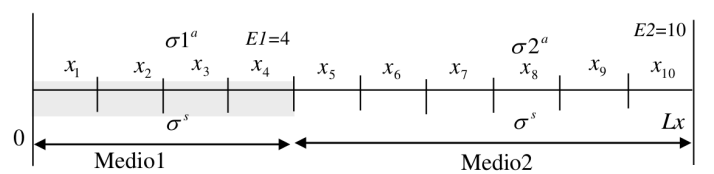

Figura 10: Geometría para el cálculo de los coeficientes de dos medios.

La Fig. 11(arriba), muestra los errores porcentuales obtenidos al estimar los coeficientes de absorción y esparcimiento para 7 procesos de iteración. Se observa que los errores porcentuales disminuyen. La Fig. 11(medio) representa los resultados obtenidos para el coeficiente de absorción y esparcimiento, variando el medio 2 , entre [0.1-1.0], el coeficiente de absorción en el medio 1 se mantiene en 0.3 y el coeficiente de esparcimiento en 0.5. La Fig. 11(abajo) muestra los resultados en la estimación del coeficiente de absorción obtenidos en 10 casos con ruido aleatorio hasta 
de $1 \%$. La placa 1 tiene $\sigma=0.3$ y la placa $2 \sigma^{a}=0.7$, en ambas placas $\sigma^{s}=0.5$.
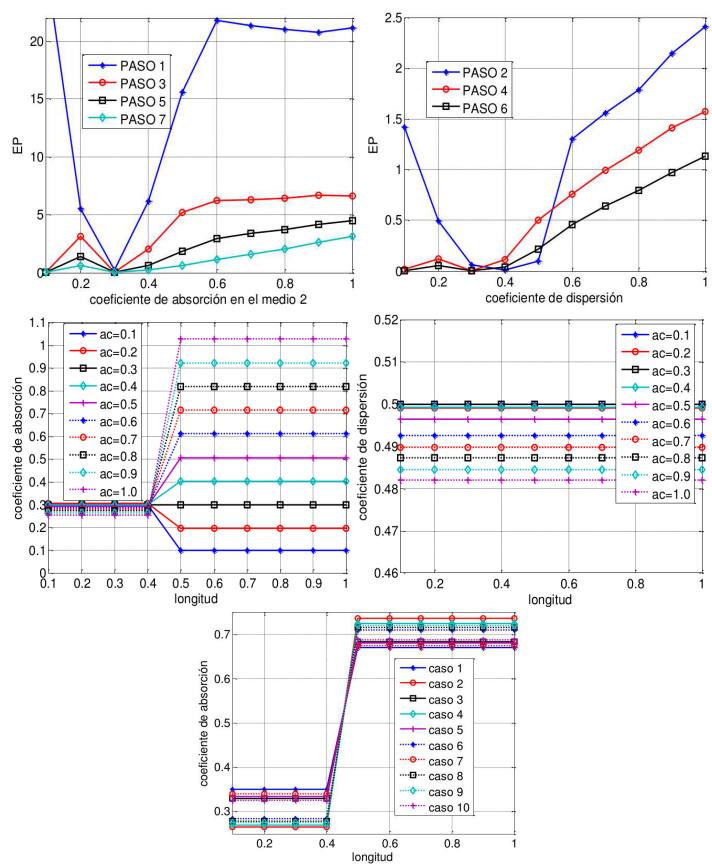

Figura 11: Resultados con ruido aleatorio hasta el $1 \%$.

\section{Conclusiones}

El empleo de la distancia de Bregman construída con la funcional relacionada a la entropía de Havdra-Charvát proporciona un conjunto de resultados para cada parámetro de $r$. Encontramos que, cuando no se tiene un conocimiento previo de los valores a ser estimados, un valor óptimo para la estimación de del coeficinte de absorción es $r=10$ y para la estimación del coeficiente de esparcimiento, $r=6$. Usando en ambos casos como valor inicial del coeficiente respectivo $10^{-6}$.

En el caso que se tenga un conocimiento previo de los posibles valores de $\sigma^{a}$ y $\sigma^{s}$, el valor óptimo del parámetro $r$ para la estimacións de los coeficientes de absorción y esparcimiento es $r=4$. Esta información es empleada como estrategia y descrita en los procesos de iteración descritos en la sección anterior. Los procesos iterativos 1-4 son los más importantes y la repetición de los procesos 3 y 4 ayuda a mejorar nuestras estimaciones.

En un trabajo futuro pretendemos expandir el problema unidimensional para la estimación de $\sigma^{a}$ y $\sigma^{s}$ en un medio bidimensional.

\section{Agradecimientos}

M. J. Berrocal Tito y R. F. Carita Montero agradecen al profesor N. C. Roberty por las discusiones acerca del presente trabajo.

\section{Referencias}

[1] G. T. Herman, Image Reconstruction from Projections: The Fundamentals of Computerized Tomography, Academic Press (1980).

[2] F. Natterer y F. Wubbeling, Mathematical Methods Image reconstruction, segunda edición, SIAM, Philadelphia (2001).

[3] F. M. Modest, Radioative Heat Transfer, McGraw-Hill, Inc., New York (2013).

[4] K. D. Lathrop, Nuclear Sci. and Eng. 32, 357 (1968).

[5] J. C. Chai, H. S. Lee y S. V. Pantakar; Numerical Heat Transfer 24(B), 373 (1993).
[6] M. J. Berrocal Tito, N. C. Roberty, A. J. Silva Neto, J. B. Cabrejos; Anais V EMC 2002, pp. 284-294 (2002).

[7] L. M. Bregman, USSR Comp. and Math. Phys. J. 7, 200 (1967).

[8] J. Havdra y F. Charvat; Kybernetika 3, 30 (1967).

[9] S. Chandrasekhar, Radiative Transfer, Dover Publications, New York (1960).

[10] W. A. Fiveland, J. Heat transfer 109, 809 (1987).

[11] C. E. Shannon, Bell System Tech. J. 27, 379 (1948). 